بعض الطرائق غير الكيميائية في مكافحة مرض القشرة السوداء على البطاطا المتسبب عن الفطر Rhizoctonia solani

\author{
عدي نجم اسماعيل مطني \\ قسم وقاية النبات- كلية الزر اعة/ جامعة بغداد
}

Email: Oadi77@yahoo.com

\title{
الخلاصة
}

نفذت هذه الدراسة في احد الحقول التنابعة لقسم وقاية النبات_كلية الزراعة/جامعة بغداد للموسم الربيعي 2010-

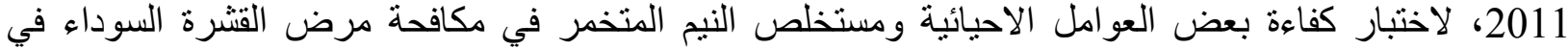

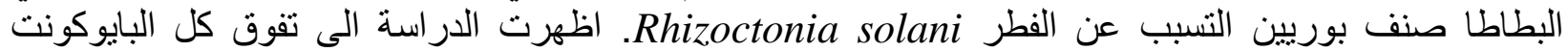
و الخميره Rhodotorula sp و S.cerevisia ومستخلص النيم المتخمر و البكتريا

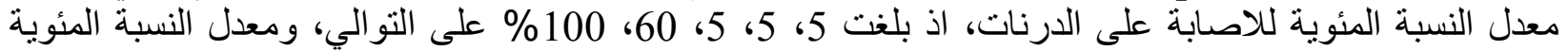

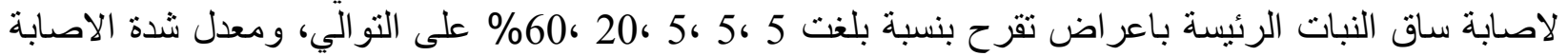

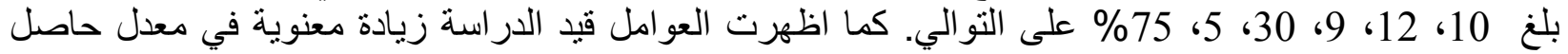

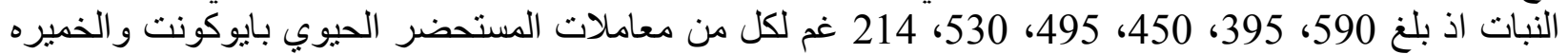
Rhodotorula sp و و S.cerevisia

كلمات دالة: مرض القشرة السوداء، بطاطا، Rhizoctonia solani، مكافحة.

تاريخ تسلم البحث 5/15 /2013 وقبوله 9 / 9 /2013

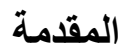

يعد الفطر Rhizoctonia solani من فطريات التربة الممرضة والمنتشرة في معظم بلدان العالم ويعيش في الإي

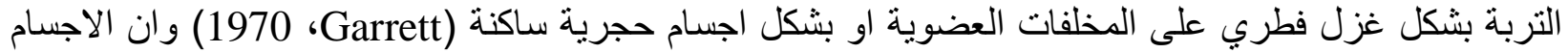

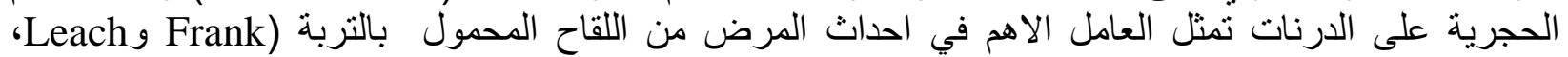

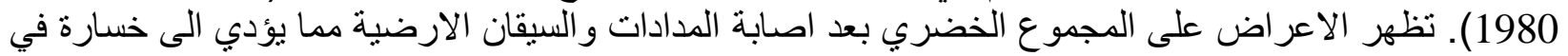

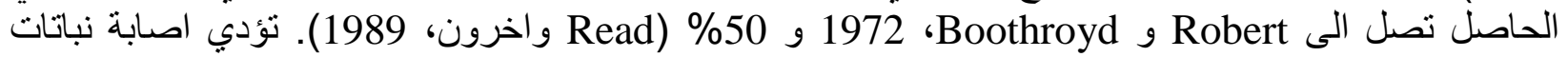

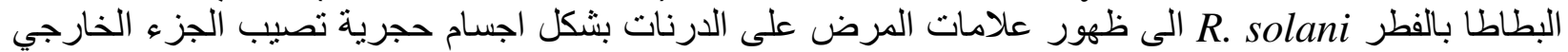

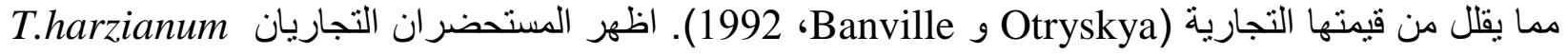

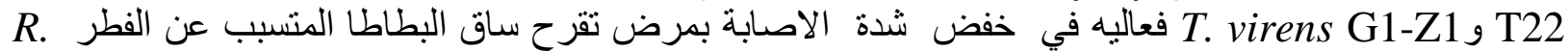
solani

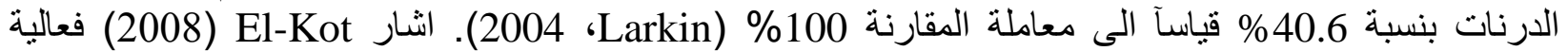

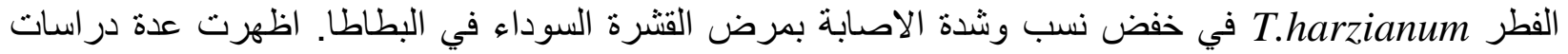

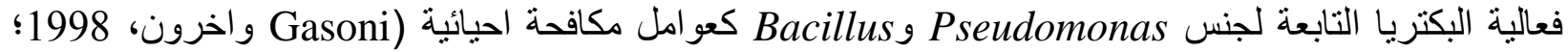
Altindag Pseudomonas

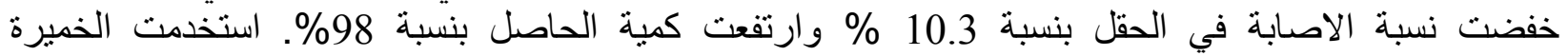

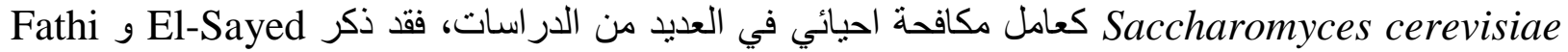

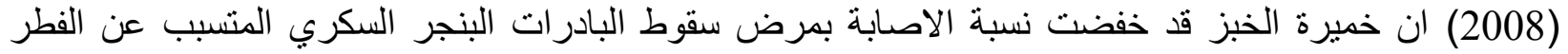

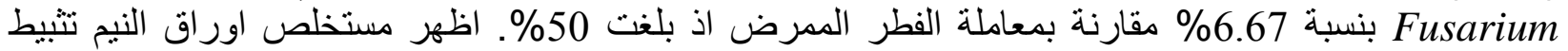

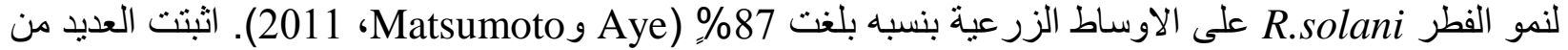
البحوث و الدراسات كفاءة الخميرة Rhodotorula spp كعامل مكافحة الحيائي ضد العد العديد من المسببات المرضية

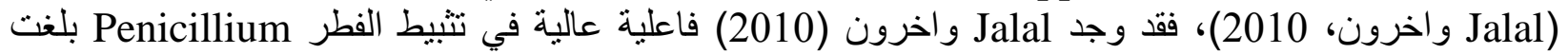

وقائع المؤتمر الدولي الثاني لعلوم وقاية النبات 19- 20 تثرين الثاني 2013 كلية الزراعة و الغابات / جامعة الموصل 


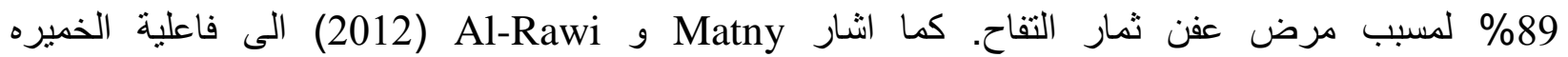
Rhodotorula spp في تثبيط النمو على الوسط الزرعي بنسبة بلغت 70\%

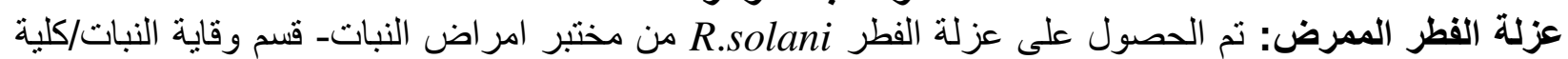

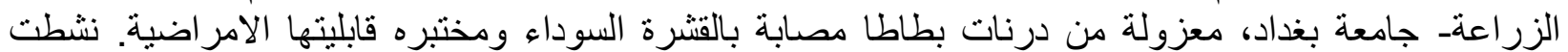
العزلة على وسط مستخلص البطاطا والدكستروز

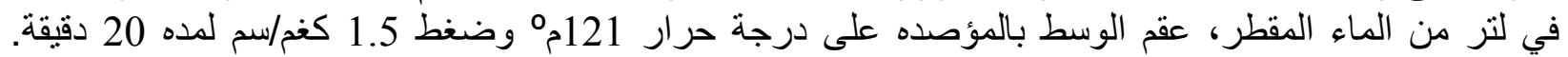

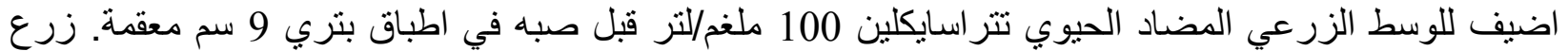

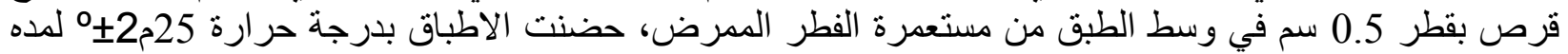
3 تحضير لقاح الفطر Rolani.sol دسل 2 كغم حبوب دخن عده مرات للتخلص من الاتربة والثو ائب العالقة، وزعت

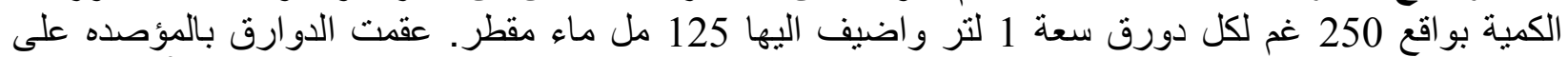

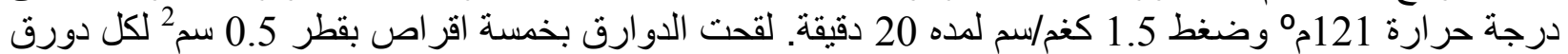

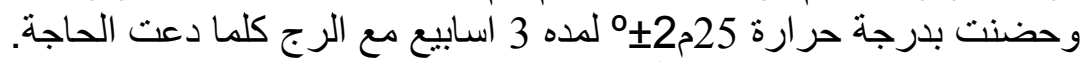

تحضير لقاح العوامل الاحيائية 1- الخميرة Saccharomyces cerevisiae: عزلت الخميره S. cereveisiae من مستحضر الخميره الخيره التجاري

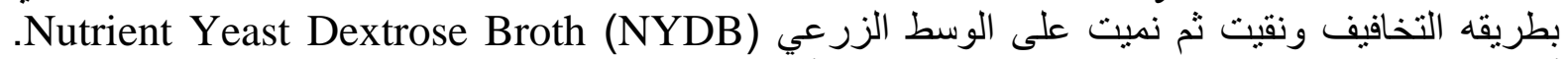

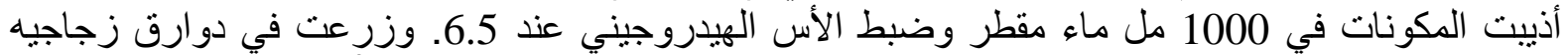

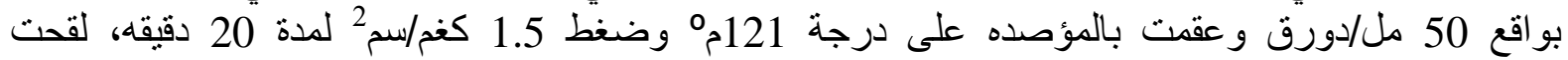
الدوارق بالخميره S.cerevisiae بو اقع 1مل/دورق من وسط مستعدرة الخميرة وحضنت في درجة حراره

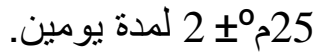

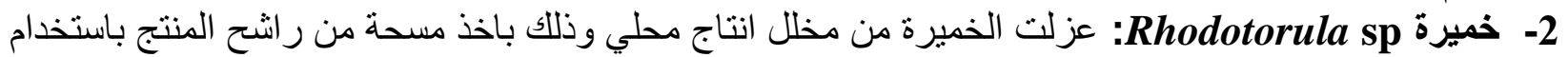

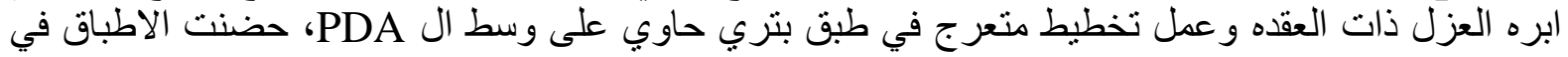

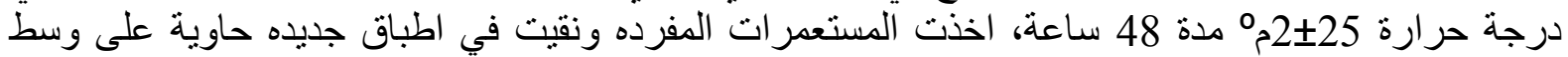

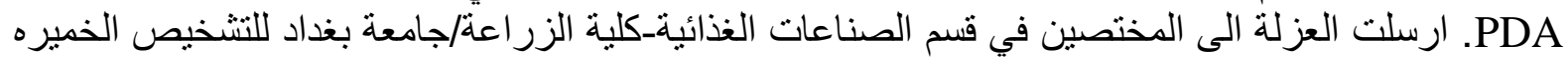

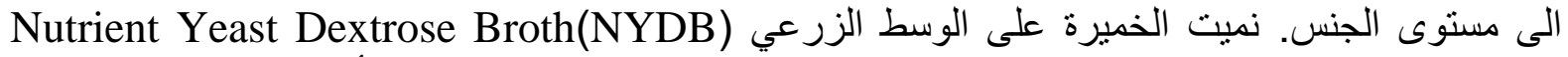

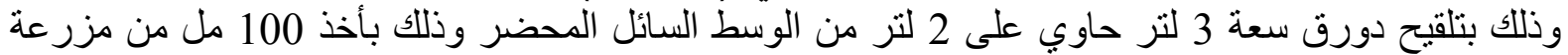
خميرة منشطة سابقا على نفس الوسط بعمر 28 ساعة، حضن الدورق في درجة حرارة 25د2مه لمدة 48

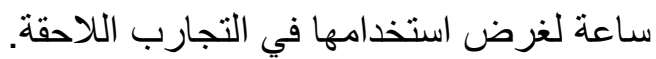

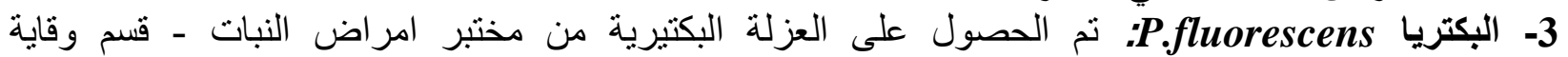
النبات/كلية الزر اعة- جامعة بغداد. حضر وسط المرق المغذي البياء

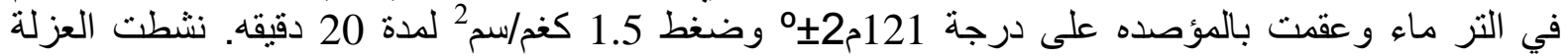
البكتيرية في 100 مل من وسط NB باخذ عده مسحات من البكتريا المنماه على وسط

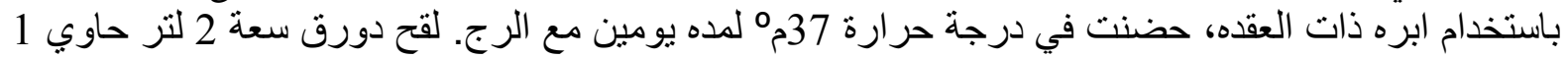

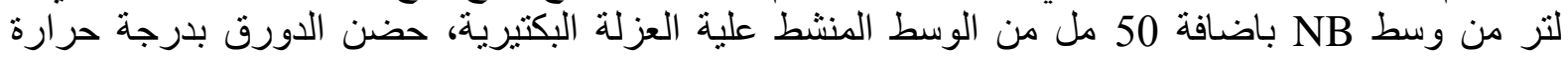
37 مج لمده يومين.

4- الفطر T.harzianum: تم الحصول على العامل الاحيائي من السوق التجارية بايوكونت انتاج شركة الرؤيا

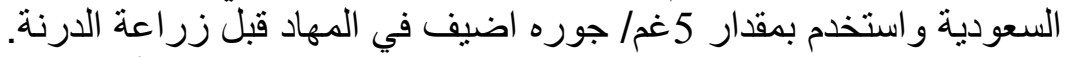

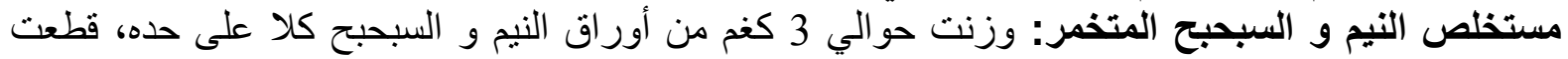

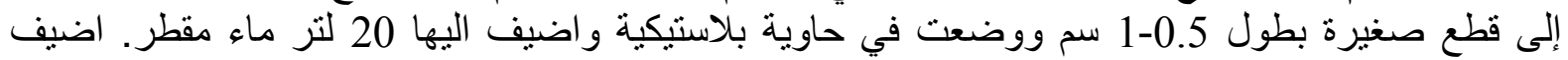
450 مل من معلق الكائنات الحية الدقيقة الفعالة (Effective Microorganisms) المنتج من قبل شركة

وقائع المؤتمر الدولي الثاني لعلوم وقاية النبات 19- 20 تشرين الثاني 2013 كلية الزراعة و الغابات / جامعة الموصل 
EMRO-CO اليابان، و 450 مل من المولاس. وضعت الحاوية البلاستيكية في درجة حرارة المختبر مده

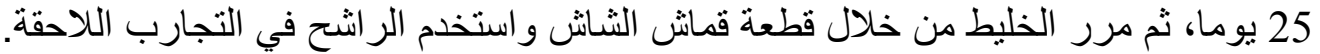

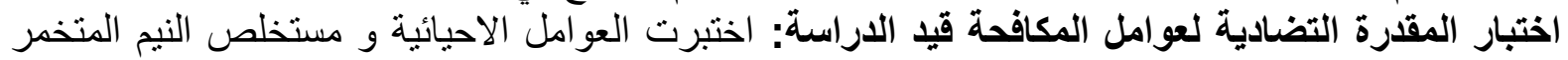

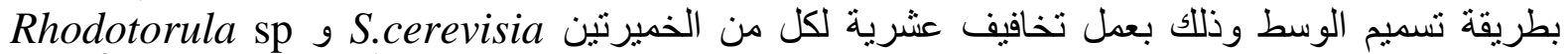

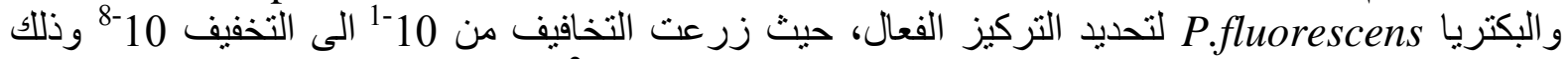

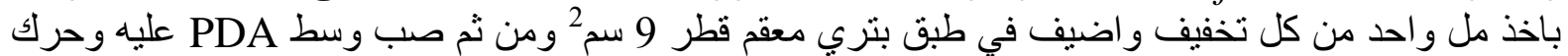

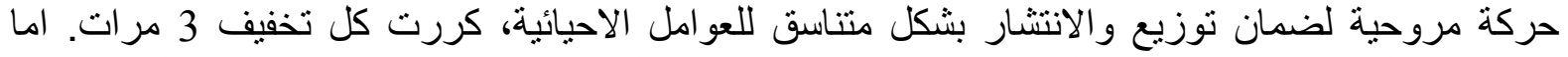

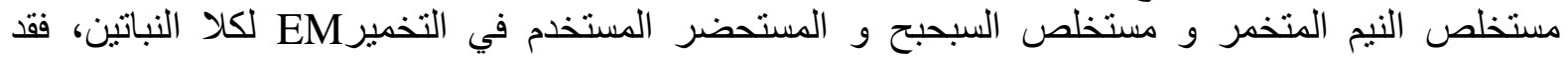

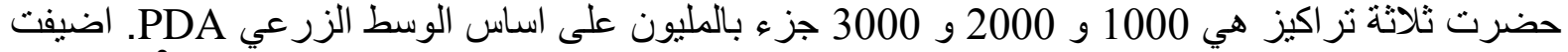

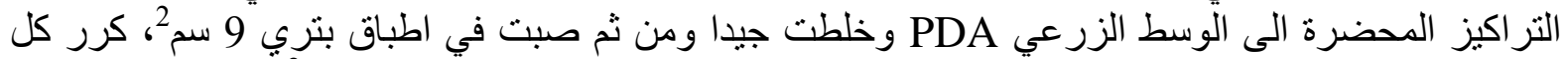

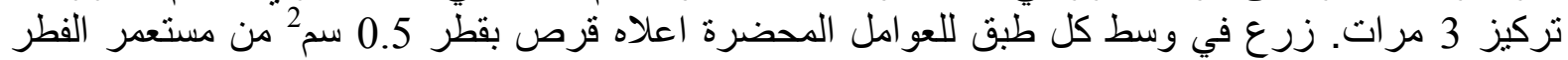
R.solani

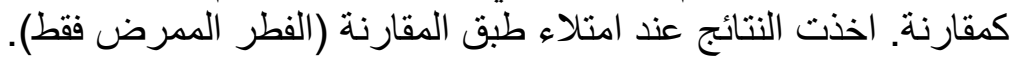

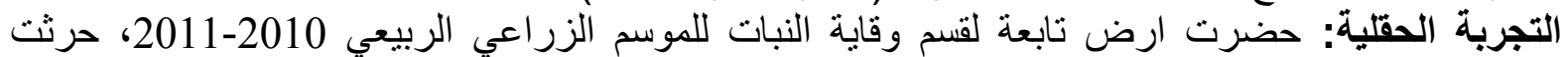

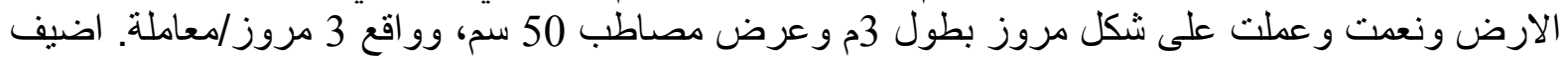

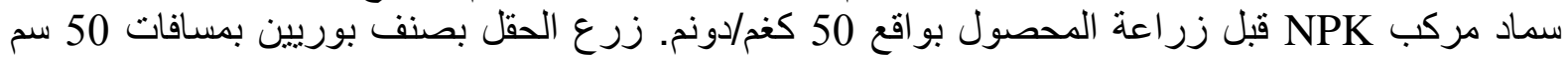

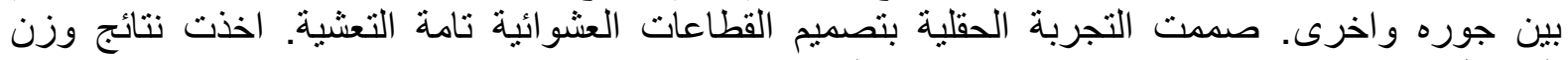
الحاصل و نسبة الاصابة وشده الاصابة في نهاية الموسم.

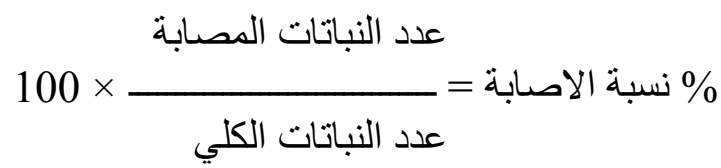

حسبت شدة الاصابة بالمرض على السيقان باستعمال الدليل المرضي المقترح من قبل( Hall واخرون (2001) مع بعض التحوير من قبل الباحث:

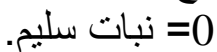
1= بقعة واحدة قطر ها اقل من 25 ملم.

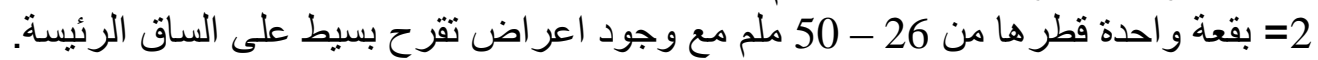
3= وجود مجموعة بقع قطر ها اكثر من 50 ملم مع وجود اعر اض تقر تقرح على الساق الرئيسة بنسبة اكثر من $\% 25$ 4= وجود مجموعة بقع قطرها اكثر من 50 ملم مع وجود اعراض تقرح على الساق الرئيسة بنسبة اقل من $\% 50$ 5= 5\% وجود مجمو عة بقع قطر ها اكثر من 50 ملم مع وجود اعر اض تقرح على الساق الرئيسة بنسبة اكثر من $\% 50$ حسبت النسبة المئوية لثدة الاصابة بالاعتماد على معادلة Mckinney (1923) وكما ياتي:

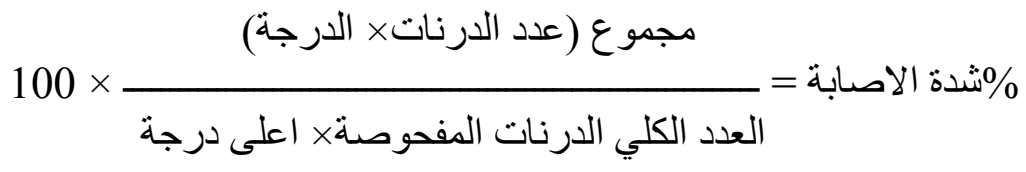

حلات النتائج احصائيا وفق تصميم القطاعات العشوائية الكاملة CRBD باستخدام البرنامج الاحصائي GenStat Discovery Edition 3 
معاملات التجربة الحقلية: اضيفت عوامل المكافحة الاحيائية بتغطيس درنات البطاطا بمعلق البكتريا

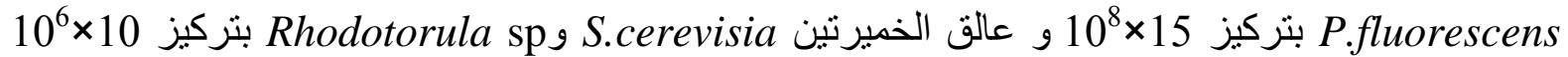

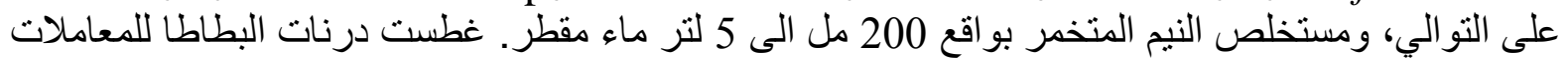

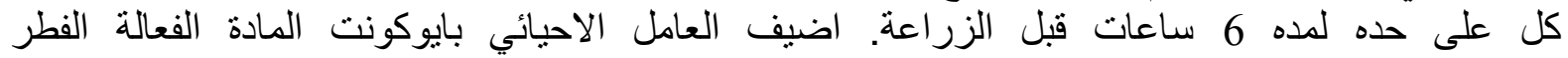

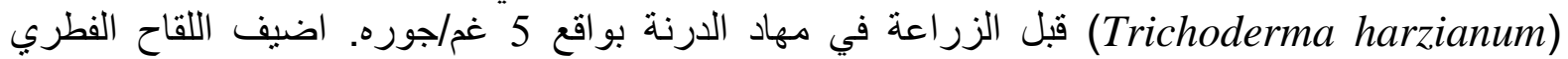

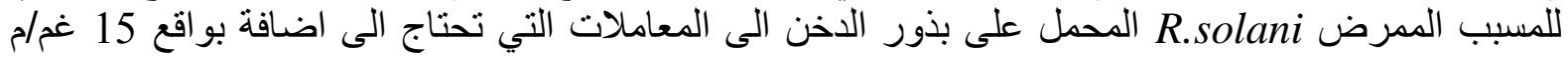

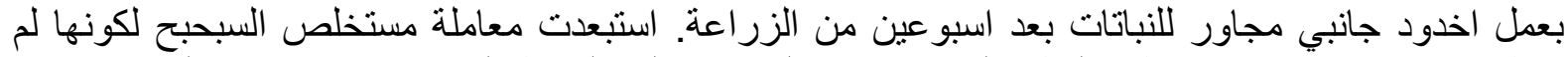

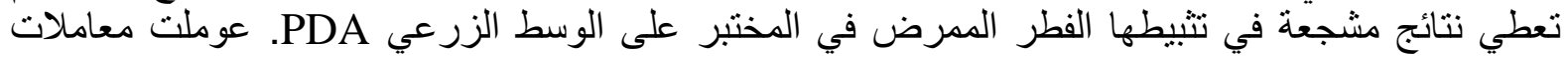

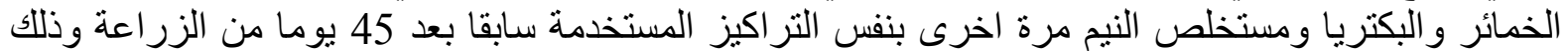

برش المنطقة التاجية للنباتات بالتر اكيز المحضرة.

$$
\begin{aligned}
& \text { 1. فطر ممرض فقط. } \\
& \text { 2. مقارنة (بدون فطر ممرض). }
\end{aligned}
$$

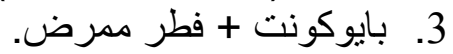$$
\text { 4. 1 بايوكونت فقط. }
$$

6

S.cerevisia .6

Rhodotorula sp .7

R 8 .

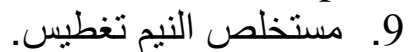

10. مستخلص النيم تغطيس+ فطر ممرض.

P.fluorescens .11

P.fluorescens.12 تغطيس فطر ممرض.

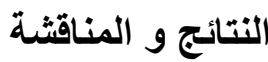

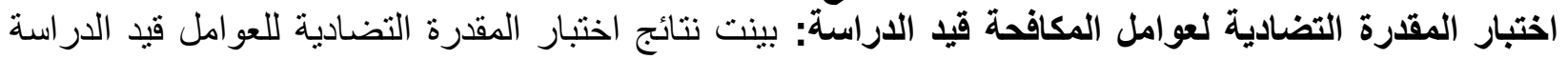

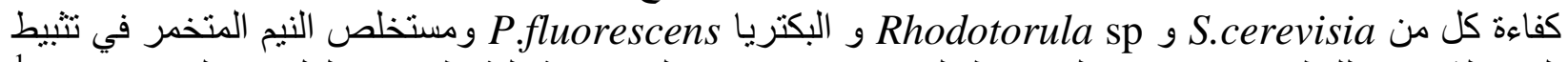

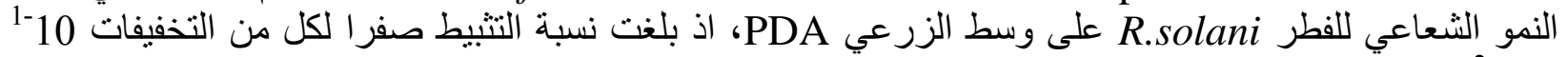

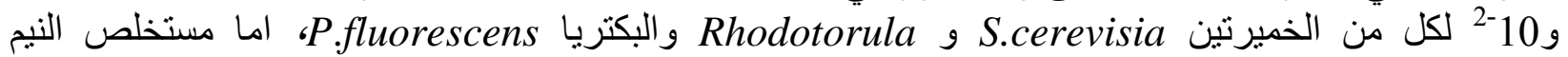

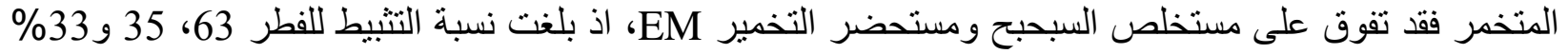

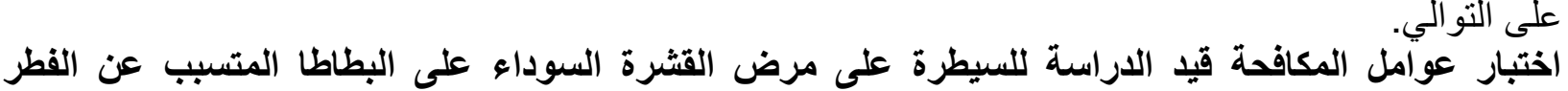

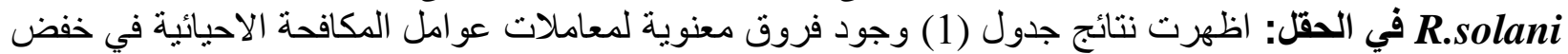

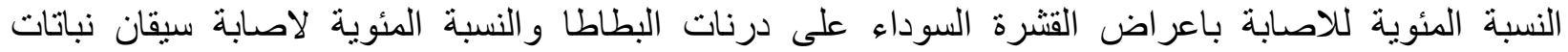

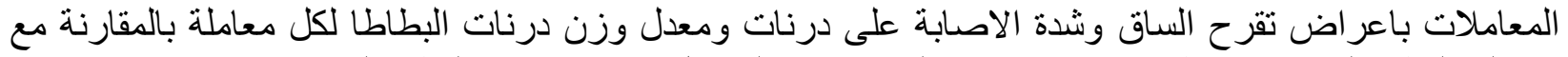

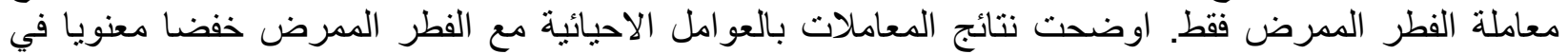

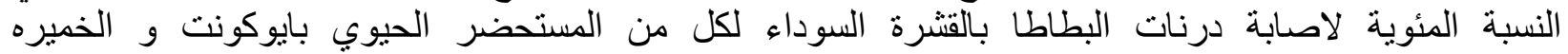
Rhodotorula sp و البكتريا S.cerevisia

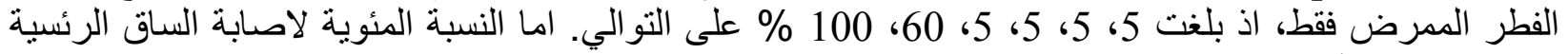

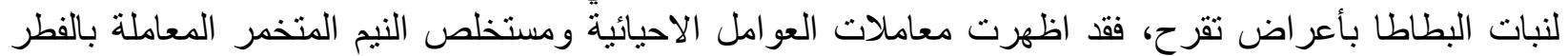

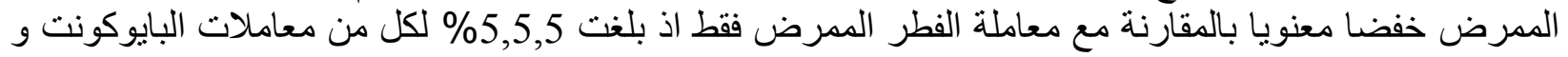

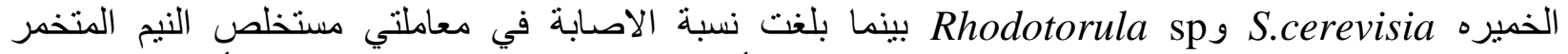

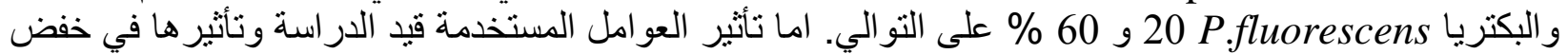

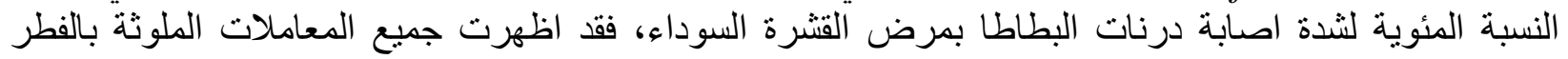

وقائع المؤتمر الدولي الثاني لعلوم وقاية النبات 19- 20 تثرين الثاني 2013 كلية الزراعة و الغابات / جامعة الموصل 
الممرض و المعاملات المعاملة بعو امل المكافحة مقدرة واضحة في خفض معدل شدة الاصابة على الدرنات الدات وبفارق

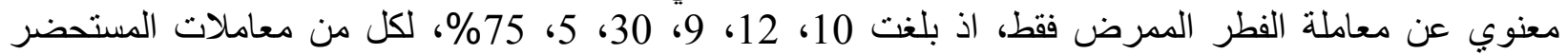
الحيوي بايوكونت

R. solani الجدول (1): تاثثر عوامل المكافحة قيد الدراسة في مكافحة مرض القثرة السوداء المتسبب غن الفطر في البطاطا.

Table (1): Effect of control agents to control black scarf disease caused by R.solani on potato.

\begin{tabular}{|c|c|c|c|c|}
\hline $\begin{array}{c}\text { درندات وزن البطاطا/غم } \\
\text { Mean } \\
\text { Meight of } \\
\text { potato } \\
\text { tubers /g }\end{array}$ & $\begin{array}{c}\text { الاصنابة لثدة } \\
\text { \% الثداتة } \\
\text { \% Disease } \\
\text { severity } \\
\text { on tuber }\end{array}$ & $\begin{array}{c}\text { \% الإقـابة } \\
\text { \%Disease } \\
\text { incidence } \\
\text { on stem }\end{array}$ & $\begin{array}{c}\text { \% الدرنات } \\
\text { \%Disease } \\
\text { incidence } \\
\text { on tuber }\end{array}$ & $\begin{array}{c}\text { المعاملات } \\
\text { Treatments }\end{array}$ \\
\hline 214 & 75 & 100 & 100 & 1. فطر ممرض فقط Pathogen only \\
\hline 406 & 0 & 0 & 0 & 2. مقارنة (بدون فطر ممرض) Control \\
\hline 590 & 10 & 5 & 5 & $\begin{array}{r}\text { 3. بايوكونت + فطر ممرض } \text { Biocont+Pathogen } \\
\text { Biogen }\end{array}$ \\
\hline 430 & 0 & 0 & 0 & 4. بايوكونت فقط Biocont only \\
\hline 395 & 12 & 5 & 5 & 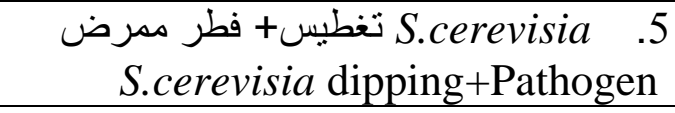 \\
\hline 430 & 0 & 0 & 0 & $\begin{array}{r}\text { S.cerevisia } .6 \\
\text { S.cerevisia dipping only }\end{array}$ \\
\hline 420 & 0 & 0 & 0 & $\begin{array}{r}\text { Rhodotorula spp } \\
\text { Rhodotorula spp dipping only } \\
\end{array}$ \\
\hline 450 & 9 & 5 & 5 & 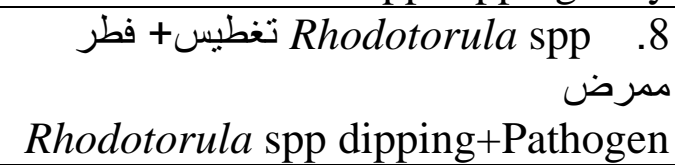 \\
\hline 375 & 0 & 0 & 0 & 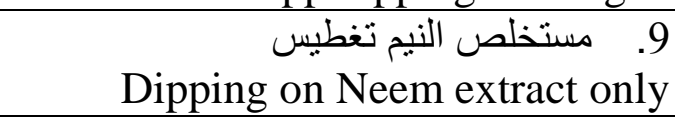 \\
\hline 495 & 5 & 20 & 5 & $\begin{array}{r}\text { 10. مستخلص النيم تغطيس + فطر ممرض extract dipping+Pathogen } \\
\text { Neem }\end{array}$ \\
\hline 400 & 0 & 0 & 0 & $\begin{array}{r}\text { تغطيس P.fluorescens .11 } \\
\text { Dipping on P.fluorescens only }\end{array}$ \\
\hline 530 & 30 & 60 & 60 & $\begin{array}{l}\text { 12. تغطيس+ فطر ممرض P.fluorescens } \\
\text { Dipping on P.fluorescens+Pathogen }\end{array}$ \\
\hline 160 & 17 & 20 & 15 & LSD 0.05 \\
\hline
\end{tabular}

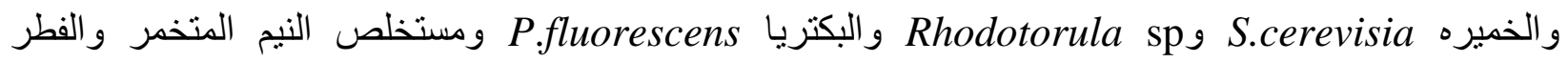

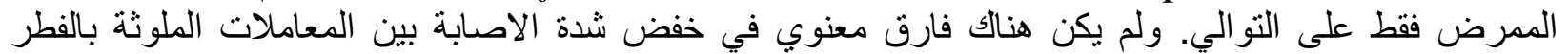

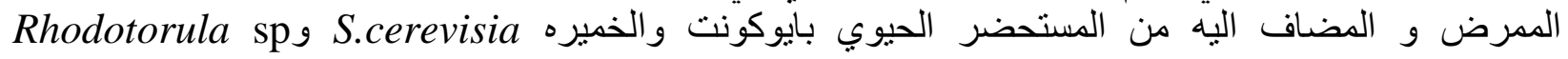
وقائع المؤتمر الدولي الثاني لعلوم وقاية النبات 19- 20 تثرين الثاني 2013 كلية الزراعة و الغابات / جامعة الموصل 


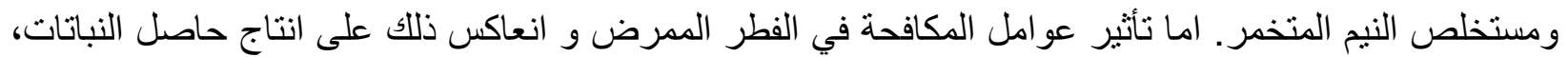

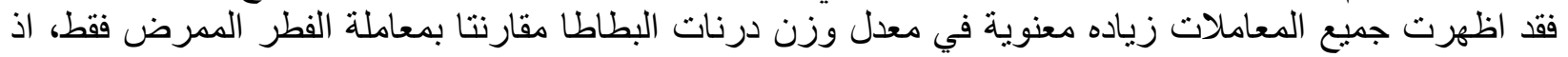

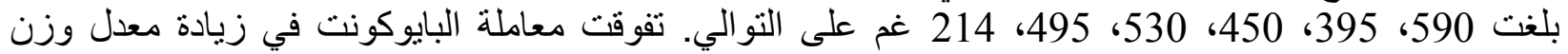

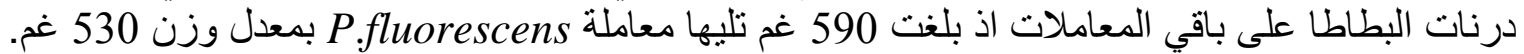

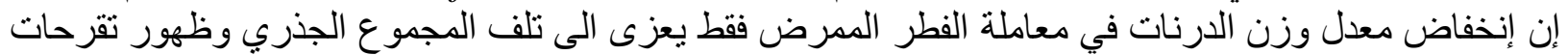

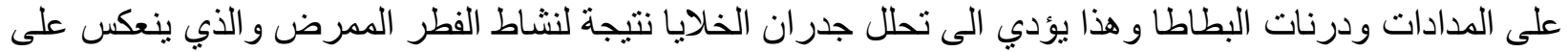

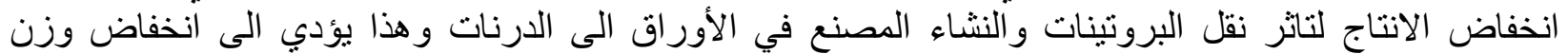

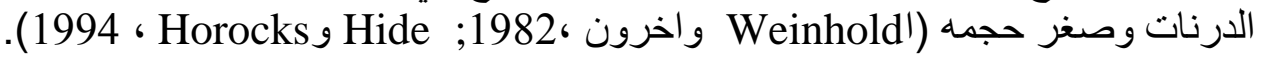

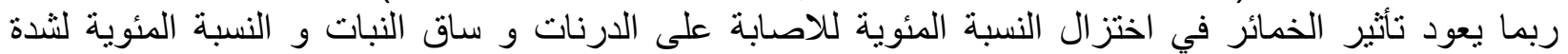

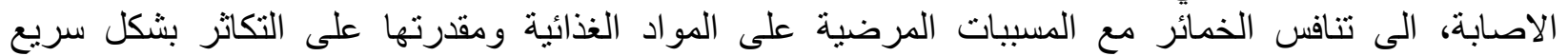

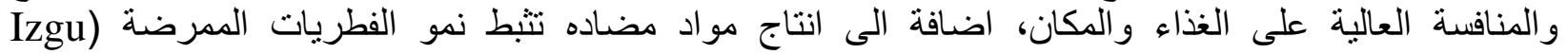

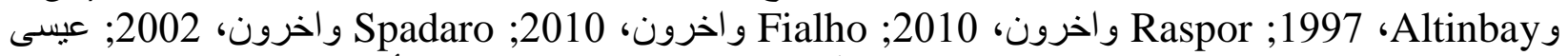

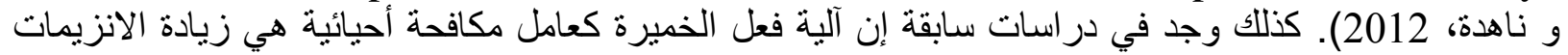
المتعلقة بالمقاومة الجهازية في ألنبات مثل انزيم 2000، Attyia 2000-Sayed) Trichoderma spp

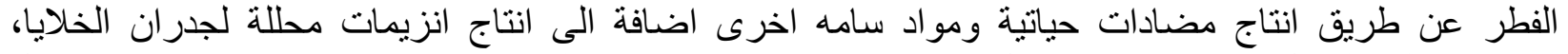

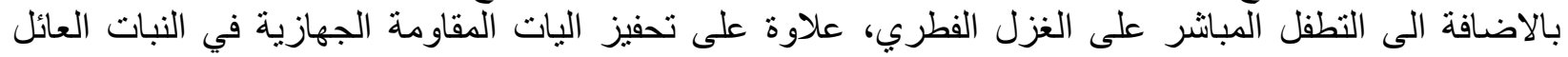
Enkerly)

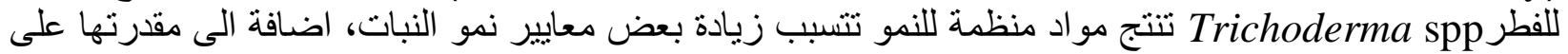

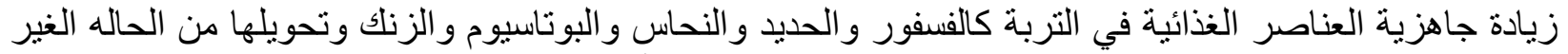

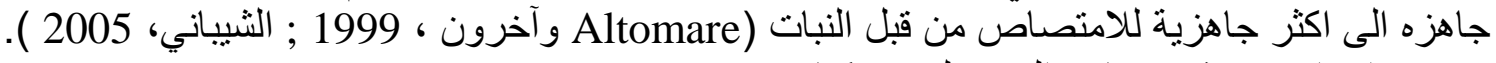

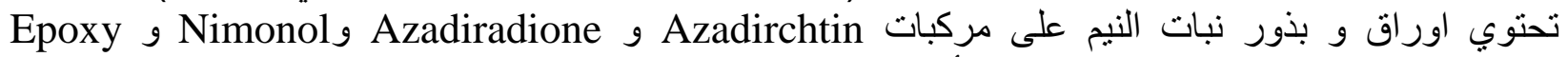
azadradion

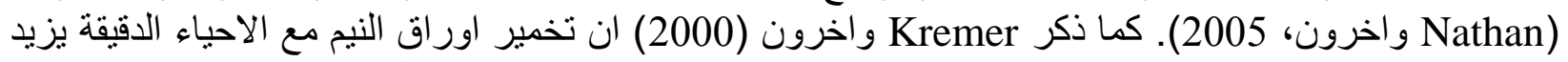

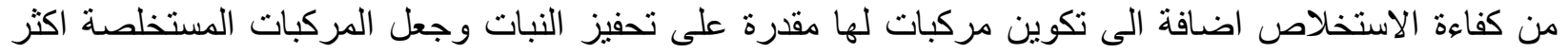

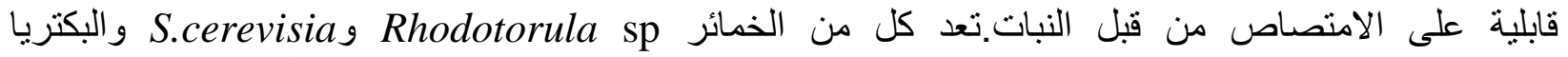

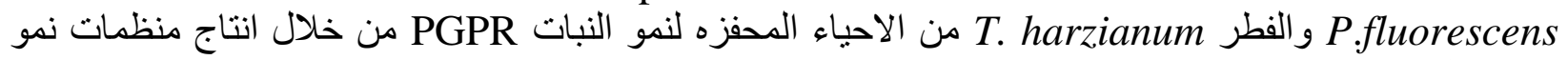
اضافة الى زياده جاهزية العناصر الغذائية للنبات (Van Loon والاحير Van واخرون، 1998).

\section{SOME NON CHEMICAL METHODS TO CONTROL BLACK SCURF DISEASE OF POTATO CAUSED BY Rhizoctonia solani}

Oadi N. Matny

Department of Plant Protection, College of Agriculture, University of Baghdad.

Email: Oadi77@yahoo.com

\section{ABSTRACT}

This study was carried out in the fields of Plant Protection Department-College of Agriculture/University of Baghdad, during spring season 2010-2011, to test the efficiency of some biological factors and fermented neem extract to control black scurf disease caused by Rhizoctonia solani in Burien potato cultivar.The study showed the superiority of biological agent Biocont (Trichoderma harzianum) and the yeasts S.cerevisia and Rhodotorula $s p$ and the fermented neem extract and bacteria P.fluorescens in reducing

$$
\text { وقائع المؤتمر الدولي الثاني لعلوم وقاية النبات 19- } 20 \text { تثرين الثاني } 2013 \text { كلية الزراعة و الغابات / جامعة الموصل }
$$


the percent infection of tubers, as amounting to 5, 5, 5, 60 and $100 \%$ respectively, rate of the canker symptoms in the main stem of plants, it was 5, 5, 5, 20 and $60 \%$ respectively, and disease severity on tubers at 10,12, 9,30,5 and $75 \%$, respectively. All tested agents showed significant increase in the rate of yield/plant, which was recoreded at 590, 395, 450, 495, 530 and $214 \mathrm{~g}$ each to the treatments Biocont and yeasts S.cerevisia, Rhodotorula sp and the bacteria P.fluorescens and fermented neem extract and the pathogen (R.solani only), respectively.

Keywords: Black scarf disease, Potato, Rhizoctonia solani, Control.

Received 15/5/2013 Accepted 9/9/2013

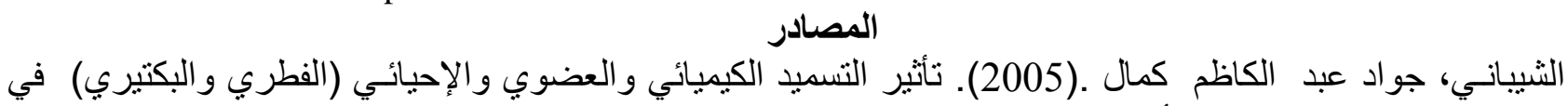

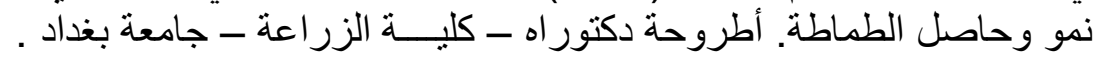

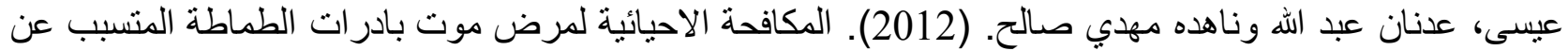

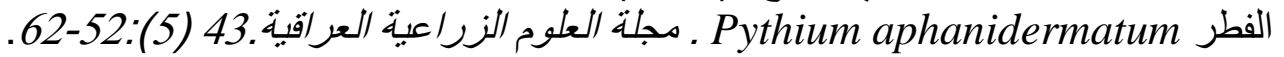

Altindag, M., Sahin, M., Esitken, A., Ercisli, S., Guleryuz, M., Donmez, M.F., and Sahin, F,.(2006). Biological control of brown rot (Moniliana laxa Ehr.) on apricot (Prunus armeniaca L. cv. Hacihalilo_lu) by Bacillus, Burkholdria, and Pseudomonas application under in vitro and in vivo conditions. Biological Control 38 (3):369-372.

Altomar, C.W. Norvell, A.T. Bjorkman and G.E.Harman.(1999).Soulabilization of phosphates and micronutrient by theplant growth promoting an biocontro 1 fungus Trichoderma harzianum Rifai 1295-22. Applied Environmental Microbiology65(7):2926-2933.

Attyia, S.H.and A.A. Youssry.(2001). Application of Saccharomyces cerevisia as a biocontrol agent against some diseases of solanaceae caused by Macrophomina phaseolina and Fusarium solani . Egyptian Journal of Biology,3:79-87.

Aye, S.S., and M Matsumoto. (2011). Effect of some plant extracts on Rhizoctonia spp. and Sclerotium hydrophilum. Journal of Medicinal Plants Research. 5(16):3751-3757.

El-Kot, G.A.N..(2008). Biological control of black scurf and dry rot of potato. Egypt Jornal of Phytopathology. 36(1-2): 45-56.

El-Sayed S., M., M Fathi El-Nady.(2008). Application of Saccharomyces cerevisiae as a biocontrol agent against Fusarium infection of sugar beet plants. Acta Biologica Szegediensis. 52(2):271-275.

El-Sayed, S.(2000).Microbial agents as a plant growth promoting and root protector $.10^{\text {th }}$.Microbiology Conference.12-14 Nov. Cairo.Egypt,p.120

Enkerly, J., G. Felix and T. Boller, .(1999). Elicitor activity of fungal xylanase does not depend on enzymatic activity. Journal of Plant Physiology, 121:391-398.

Fialho, M.B., L. Toffano., M.P. Pedroso., F. Augusto and S.F. Pascholati. (2010). Volatile organic compounds produced by Saccharomyces cerevisiae inhibit the in vitro development of Guignardia citricarpa the causal agent of citrus black spot. World Jornal Microbiology Biotechnol. 26:925-932.

وقائع المؤتمر الدولي الثاني لعلوم وقاية النبات 19- 20 تشرين الثاني 2013 كلية الزر اعة و الغابات / جامعة الموصل 
Frank, J. A. and Leach, S.S .(1980). Comparison of tuber borne and soilborne inoculum in the Rhizoctonia disease of Potato. Phytopathology. 70:51-53.

Garrett, S.D .(1970). Pathogenic Root-Infecting Fungi. Cambridge Univ. Press, Cambridge, England, pp 294.

Gasoni, L., Cozzi, J., Kobayashi, K., Yossen, V., Zumelzu, G., and S. Babbitt. (1998). Suppressive effect of antagonistic agents on Rhizoctonia isolates on lettuce and potato in Argentina field plots. In: International Congress of Plant Pathology. $\left(9^{\text {th }}-16\right.$ th August, 1998, Edinburgh, Scotland). p. 5.2.44.

Hall, B., K. Davies, and T. Wicks .(2001). Biological and Chemical Control of Rhizoctonia. HRDC Project PT 98036 south Australin Research and Development Institute Plant Research Center GPO Box 397. Adelatde SA 5001. pp.1-49.

Hide, G.A., and Horocks. J.K.(1994). Influence of stem canker (Rhizoctonia solani Kühn) on tuber yield, tuber size, reducing sugars and crisp colour in cv. Record. Potato Res. 37:43-49.

Izgu, F. and D. Altinbay.(1997). Killer toxins of certain yeast strains have potential growth inhibitory activity on Gram-Positive Pathogenic bacteria. Microbios, 89:15-22.

Jalal, G., H.R. Etebarian., and N. Sahebani .(2010). Biological control of apple blue mold with Candida membranifaciens and Rhodotorula mucilaginosa. African Journal of Food Science. 4(1):1-7.

Keszler, A., E. Forgacs., L. Kotali., J.A. Vizcaino., E. Monte., and I. Garcia-Acha,.(2000). Separation and identification of volatile components in the fermentation broth of Trichoderma viride by solid-phase extraction and gas chromatography-mass spectroscopy. Journal of Chromatograph Science, 38:421-424.

Kremer, R.J., E.H. Ervin., M.T Wood., and D. Abuchar.(2000). Control of Sclerotinia homoeocarpa in turf grass using effective microorganism (EM). World J. 1:16-21.

Larkin, R.P..(2004). Development of Integrated Biological and Cultural Approaches For Control of Powdery Scab and Other Soil Borne Disease. USDA, ARS, New England Plant, soil, and water lab Univer. of Maine, Orone, ME O 44469.

Lorito, M,.(1998). Chitinolytic enzymes and their genes. in: Trichoderma and Gliocladium. Vol 2. G.E. Harman and C.P. Kubicek, eds. Taylor \& Francis, London, pp:73-99.

Matny, O.N., and F.I Al-Rawi.(2012). Use of antimicrobial and biological agent to control green mold on orange fruit. International Journal of Applied Agricultural Research. $7(1): 45-54$.

Mohsin, T., S Yasmin., and F Y. Hafeez,.(2010). Biological control of potato black scurf by rhizosphere associated bacteria. Brazilian Journal of Microbiology. 41: 439-451.

Nathan, S S., K. Kalaivani and K. Murugan.(2005).Effect of neem limonoids on the malaria vector Anopheles stephensi (Diptera: Culicidae). Acta Top., 96:47-55.

Otryskya, B. E., and G. J. Banville.(1992). Effect of infection by Rhizoctonia solani on the quality of tuber for processing. American Potato. Jornal 69:645-652.

Raspor, P., D.M. Miklic., M. Avbelj., and N. Cadez .(2010). Biocontrol of grey mold disease on grape caused by Botrytis cinerea with Autochthonous wine yeasts . Food Technology Biotechnology. 48(3):336-343.

وقائع المؤتمر الدولي الثاني لعلوم وقاية النبات 19- 20 تثرين الثاني 2013 كلية الزراعة والغابات / جامعة الموصل 
Read, P., J. Hide., G.A. Firmager., and S.M. Hall .(1989). Growth and yield of Potatoes as attected by severity of stem canker (Rhizoctonia solani). Potato Res. 32:9-15.

Robert, D.A. and C.W. Boothroyd.(1972).Fundamentals of Plant Pathology.W.F. Freeman and Co. San Francisco. pp 482.

Spadaro, D., R. Vola., S. Piano., and M.L. Gullino.(2002). Mechanisms of action and efficiency of four isolates of the yeast Metschnikowia pulcherrima active against postharvest pathogens on apples. Postharvest Biological Technology 24:123-134.

Van Loon, L.C., P. Bakker., and M.J Pieters.(1998). Systemic resistance induced by rhizosphere bacteria. Annual Review of Phytopathology. 36:453-483.

Weinhold, A. R., T. Bowman., and D.L. Hall.(1982). Rhizoctonia disease of Potato effect on yield and control by seed tuber treatment. Plant Disease 66:815-818.

وقائع المؤتمر الدولي الثاني لعلوم وقاية النبات 19- 20 تشرين الثاني 2013 كلية الزر اعة والغابات / جامعة الموصل 